\title{
A New Method to Calibrate the Stellar COLOR/SURFACE-BRIGHTNESS RELATION
}

\author{
ANDREW Gould \\ Department of Astronomy, Ohio State University, 140 W. 18th Ave., Columbus, OH 43210, USA \\ gould@astronomy. ohio-state.edu
}

Received July 21, 2014; accepted August 12, 2014

\begin{abstract}
I show that the standard microlensing technique to measure the angular radius of a star using color/surface-brightness relations can be inverted, via late-time proper motion measurements, to calibrate these relations. The method is especially useful for very metal-rich stars because such stars are in short supply in the solar neighborhood where other methods are most effective, but very abundant in Galactic bulge microlensing fields. I provide a list of eight spectroscopically identified high-metallicity bulge stars with the requisite finite-source effects, seven of which will be suitable calibrators when the Giant Magellan Telescope comes on line. Many more such sources can be extracted from current and future microlensing surveys.
\end{abstract}

Key words: astrometry — gravitational microlensing — stars: fundamental parameters (radii)

\section{INTRODUCTION}

Stellar radii $R$ are fundamental parameters but are directly measured for a tiny fraction of all stars. They can, however, be accurately estimated from $R=d \theta_{*}$ if the distance $d$ is known and the angular radius $\theta_{*}$ can be determined using color/surface-brightness relations. For example, Kervella et al. (2004) give

$$
K_{0}+5 \log \left(\theta_{*} / \mathrm{mas}\right)=0.377(V-K)_{0}+1.080
$$

for dwarfs and subgiants. The left-hand side is (a logarithm of) the mean surface brightness of the star, while the right-hand side is proportional to the color.

Kervella et al. (2004) argue that there is no evidence for a dependence of this relation on metallicity over the range $-0.5<[\mathrm{Fe} / \mathrm{H}]<+0.5$. However, while their Figure 5 indeed provides no evidence for a metallicity dependence, neither does it provide strong evidence against this hypothesis. Such an effect would be expected to appear most strongly in cool metal-rich stars, for which line blanketing in the $V$ band (and other blue bands) is severe. That is, Equation (1) implicitly uses $(V-K)$ as a temperature indicator, but this indicator will be faulty if the $V$ flux is suppressed by cool metals in the atmosphere. In the Kervella et al. (2004) sample of dwarfs and subgiants, it is mainly the subgiants that are cool, and none of these is more metal-rich than $[\mathrm{Fe} / \mathrm{H}]=+0.25$. It would be useful to carry out the calibration at the high-metallicity end by including additional stars with precise $\theta_{*}$ measurements for metal-rich stars, and in particular, subgiants and giants.

To assess the difficulty of doing so from a local sample, I search the CHARM2 catalog (Richichi et al. 2005) for "red giants", defined as having radii $R>6 R_{\odot}$ and color $V-K>1.5$, with angular diameter measurements

Corresponding AUthor: A. Gould from lunar occultations, long-baseline interferometry, fine-guidance sensor, speckle interferometry, and aperture masking (with the great majority coming from the first two). I find 372 such stars of which 184 have metallicities listed in Anderson \& Francis (2012). Figure 1 shows the cumulative distribution of these metallicities.

Note that there are only four stars with $[\mathrm{Fe} / \mathrm{H}]>$ 0.16: HIP $(22729,88567,85617,100345)$ at $[\mathrm{Fe} / \mathrm{H}]$ $(0.23,0.28,0.30,0.46)$. Furthermore, the first of these has an unusably poor $(23 \%) \theta_{*}$ measurement, and the next two are pulsating variables. Thus, the highmetallicity end of the distribution is extremely poorly probed.

\section{Microlensing AND ANgular RADII}

Microlensing studies make routine use of color/surfacebrightness relations to estimate $\theta_{*}$, which they use as an intermediate step to determine the "Einstein radius" $\theta_{\mathrm{E}}$, via the relation $\theta_{\mathrm{E}}=\theta_{*} / \rho$. Here, $\rho$ is a parameter that is returned by microlensing models, essentially whenever the source is observed to cross a caustic. That is, the duration of the source-caustic-crossing time relative to the Einstein timescale $t_{\mathrm{E}}$ of the event as a whole allows to determine $\rho=\theta_{*} / \theta_{\mathrm{E}}$.

As discussed by Yoo et al. (2004), the mathematical model of the microlensing event yields the instrumental magnitudes of the source (free of blending) in several bands, usually including $V$ and $I$. One finds the offset of these values $\Delta((V-I), I)$ from the red clump (RC) centroid, using the same instrumental photometry. The dereddened color of the clump is known to be $(V-I)_{0, \mathrm{cl}}=1.06$ from the work of Bensby et al. (2013), while the dereddened magnitude $I_{0, \mathrm{cl}}$ is known as a function of field position from the study by Nataf et al. (2013). These yield $(V-I, I)_{0}=$ $(V-I, I)_{0, \mathrm{cl}}+\Delta((V-I), I)$. Then this $V / I$ photome- 


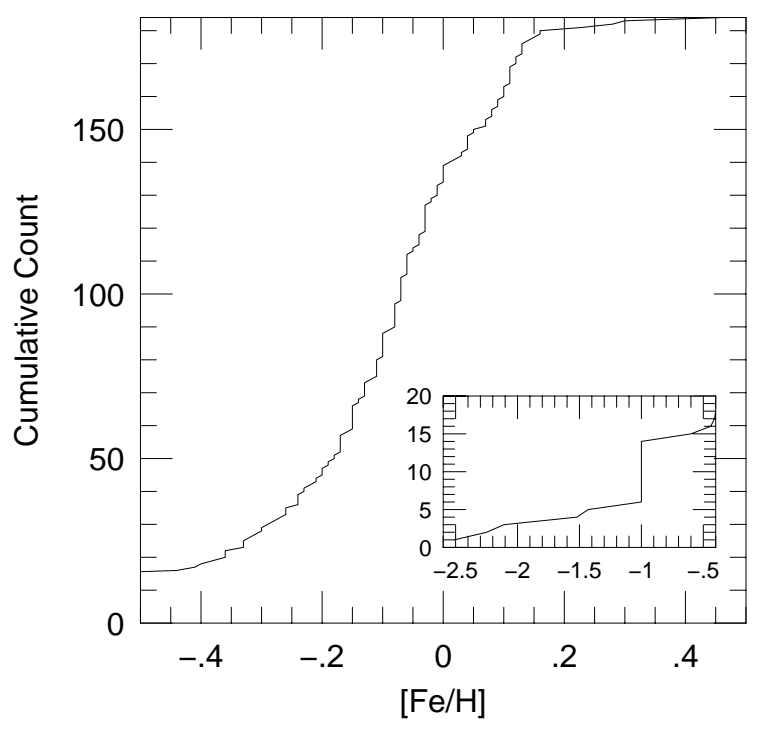

Figure 1. Cumulative distribution of "giant stars" $\left(R>6 R_{\odot}\right.$ and $V-K>1.5)$ with angular diameter measurements from Richichi et al. (2005) and metallicities from Anderson $\&$ Francis (2012). The interval $-0.3<[\mathrm{Fe} / \mathrm{H}]<0.2$ is well sampled but there are only four stars $[\mathrm{Fe} / \mathrm{H}]>0.16$.

try is converted to $V / K$ using the empirical color-color relations of Bessell \& Brett (1988). The $(V-K)_{0}$ color is used to estimate the $K$-band surface brightness employing the empirical color/surface-brightness relations of Kervella et al. (2004), and finally this is combined with $K_{0}$ to determine the source angular radius, $\theta_{*}$.

There are two principal sources of uncertainty in this estimate. First, the dereddened color is determined only to about $\sigma(V-I)_{0} \sim 0.05 \mathrm{mag}$. This uncertainty is known because the color-estimation procedure has been applied by Bensby et al. (2013) to a sample of about 50 dwarfs and subgiants with high-resolution spectra (taken when the source was highly magnified by microlensing). Then the $(V-I)_{0}$ colors were predicted from models based on spectral classification and compared to those determined by the microlensing method. For relatively blue stars near the turnoff, the scatter is about $0.06 \mathrm{mag}$, of which some contribution is due to the uncertainty in the spectroscopic temperature, implying that the intrinsic scatter in the microlensing method is $0.05 \mathrm{mag}$ (or possibly less, if there are other unrecognized errors in the spectroscopic determinations). Redder microlensed stars show greater scatter but Bensby et al. (2013) argue that this is due to uncertainty in the spectroscopic models of these stars. Note that Bensby et al. (2013) determine the color of the RC by choosing the value that minimizes this scatter.

Second, there is typically a 0.1 mag uncertainty in estimating the $I$-band magnitude of the RC centroid. These two errors combined yield a $7 \%$ error in $\theta_{*}$. No account is usually taken of errors in the overall distance scale (i.e., $R_{0}$ ) derived by Nataf et al. (2013) nor in the color/surface-brightness relations derived by Kervella et al. (2004), since these are deemed small compared to the dominant errors.

\section{Using Microlensing to Calibrate the RELATION}

The parameter $\rho$ is not always measured in microlensing events, but when it is, the above process can in principle be inverted to measure $\theta_{*}$ and so measure the surface brightness. The events for which $\rho$ is measurable are also those for which it is easiest to get a spectrum because the sources are either big (so bright) and so more likely to transit a caustic, or because the sources are highly magnified, which also makes caustic crossings more likely. Hence, these are the same stars for which it is most feasible to measure a metallicity.

The basic method is simply to wait for the source and lens to separate enough to be clearly resolved in highresolution (e.g., adaptive optics - AO) images. This automatically yields a measurement of their separation $\Delta \theta$, and hence their heliocentric proper motion

$$
\mu_{\text {hel }}=\frac{\Delta \theta}{\Delta t},
$$

where $\Delta t$ is the time interval from the peak of the event to the time of observation. Equation (2) makes two approximations. First, it assumes that the lens and source were perfectly aligned at the peak of the event. For typical events, they are misaligned by $\lesssim 100 \mu$ as, whereas $\Delta \theta$ will typically be many tens of mas (to enable separate resolution). Hence, the error induced by this approximation is usually negligible and, in any case, quantifiable. Second it ignores the lens-source parallactic motion due to their relative parallax $\pi_{\text {rel }}$. There are three points about this approximation. First, it is also typically the case that $\pi_{\text {rel }} \lesssim 100 \mu$ as, so this effect is similarly small. Second, if one were really worried about this effect, one could make the $\mathrm{AO}$ measurement at the same time of year as the peak of the event. Finally, this effect is generally smaller than one I discuss below that is also directly proportional to $\pi_{\text {rel }}$. Hence, for purposes of discussion, I simply use Equation (2) as is.

Next, if $\rho$ (and $t_{\mathrm{E}}$ ) are measured, then their product $t_{*} \equiv \rho t_{\mathrm{E}}$ is also measured. Actually, $t_{*}$ is typically measured to much higher precision than either $\rho$ or $t_{\mathrm{E}}$ separately, particularly in the high-magnification events (Yee et al. 2012). This is because $t_{*}$ reflects the caustic crossing time, which is a direct observable, whereas $\rho$ and $t_{\mathrm{E}}$ are covariant with many other parameters, including each other.

Naively, then, we have $\theta_{*}=\mu t_{*}$, and we appear to be done. Unfortunately, the caustic crossing time is measured in the frame of Earth at the peak of the event, while $\mu_{\text {hel }}$ is measured in the Sun frame, as described above. Hence, the appropriate equation is

$$
\theta_{*}=\mu_{\mathrm{geo}} t_{*},
$$

where (Janczak et al. 2010)

$$
\boldsymbol{\mu}_{\mathrm{geo}}=\boldsymbol{\mu}_{\mathrm{hel}}-\boldsymbol{\mu}_{\oplus} \pi_{\mathrm{rel}} ; \quad \boldsymbol{\mu}_{\oplus} \equiv \frac{\mathbf{v}_{\oplus, \perp}}{\mathrm{AU}}
$$


$\pi_{\text {rel }} \equiv \mathrm{AU}\left(D_{L}^{-1}-D_{S}^{-1}\right)$ is the lens-source relative parallax (with $D_{L}$ and $D_{S}$ being the distances to the lens and source, respectively), and $\mathbf{v}_{\oplus, \perp}$ is the transverse velocity of Earth in the frame of the Sun at the peak of the event.

\subsection{Uncertainty of $\theta_{*}$ Measurement}

Of course, $\boldsymbol{\mu}_{\oplus}$ is known with extremely high precision, but $\pi_{\text {rel }}$ may not be known very well, and this can lead to significant uncertainty in $\theta_{*}$ even if $\mu_{\text {hel }}$ and $t_{*}$ are well measured. To gain a sense of this, I note that the great majority of usable microlensing events peak within 2 months of opposition and also that it is only the component of $\boldsymbol{\mu}_{\oplus}$ that is aligned with $\boldsymbol{\mu}_{\text {hel }}$ that plays a significant role in Equation (4). Therefore, I adopt $\boldsymbol{\mu}_{\oplus} \cdot \boldsymbol{\mu}_{\text {hel }} / \mu_{\text {hel }}=3.5 \mathrm{yr}^{-1}$ as a typical value. In this case, an uncertainty in $\pi_{\text {rel }}$ of $100 \mu$ as leads to an uncertainty in $\mu_{\text {hel }}$ of 0.35 mas yr $^{-1}$, which should be compared to typical values of $\mu_{\text {hel }}$ of 4 mas yr $^{-1}$ and $7 \mathrm{mas} \mathrm{yr}^{-1}$ for bulge and disk lenses respectively. Hence, uncertainties in $\pi_{\text {rel }}$ must be minimized.

There are two main routes to doing so. First, since the lens is resolved, the same high-resolution images that measure its position can also be used to measure its color and magnitude, and from this one can estimate a photometric distance. Given the fact that the distribution of the dust along the line of sight is not very well known and that the photometry is likely to be mostly in the infrared, such an estimate, by itself, would be fairly crude. However, there is an additional constraint on the lens mass $M$ and the relative parallax $\pi_{\text {rel }}$ from (e.g., Gould 2000)

$$
M \pi_{\mathrm{rel}}=\frac{\theta_{\mathrm{E}}^{2}}{\kappa}=\frac{\left(\mu_{\mathrm{geo}} t_{\mathrm{E}}\right)^{2}}{\kappa} ; \quad \kappa \equiv \frac{4 G}{c^{2} \mathrm{AU}} \simeq 8.1 \frac{\mathrm{mas}}{M_{\odot}} .
$$

The combination of photometric and $\theta_{\mathrm{E}}$ constraints can be extremely powerful. For example, in a majority of cases, the lens will be in the Galactic bulge. It will therefore be behind essentially all the dust, allowing precise dereddening of the photometry. Moreover, once constrained to being in the bulge, even very large relative changes in $\pi_{\text {rel }}=\pi_{l}-\pi_{s}$ lead to small changes in distance, so that both the absolute magnitude and color can be estimated quite precisely. Then, even with considerable uncertainty in the stellar absolutemagnitude/mass relation, $\pi_{\text {rel }}$ can be estimated very precisely (e.g., Batista et al. 2014). As an example, consider an $\mathrm{M}$ dwarf lens $M=0.5 M_{\odot}$, with $\pi_{s}=110 \mu$ as and $\pi_{l}=140 \mu \mathrm{as}$, so $\theta_{\mathrm{E}}=0.35$ mas. Now, $\theta_{\mathrm{E}}$ will be known with few percent precision, so if $M$ were estimated photometrically to 20\%, then Equation (5) would lead to a $\sim 30 \%$ error in $\pi_{\text {rel }}$. However, since $\pi_{\text {rel }}$ is only $30 \mu$ as, this would propagate to only a $\sim 1 \%$ error in $\theta_{*}$.

The real difficulties posed by uncertainty in $\pi_{\text {rel }}$ come for disk lenses, which are the minority. For these, the extinction is uncertain while modest fractional errors in the lens-mass estimate, (leading to modest fractional errors in $\pi_{\text {rel }}$ ) still yield relatively large absolute errors in $\pi_{\text {rel }}$ (and so $\theta_{*}$ ) simply because $\pi_{\text {rel }}$ is itself relatively large. However, for disk lenses there is often another source of information: the microlens parallax vector $\boldsymbol{\pi}_{\mathrm{E}}$,

$$
\boldsymbol{\pi}_{\mathrm{E}} \equiv \frac{\pi_{\mathrm{rel}}}{\theta_{\mathrm{E}}} \frac{\boldsymbol{\mu}}{\mu}
$$

This quantity parameterizes the lens-source displacement due to reflex motion of Earth (though $\pi_{\text {rel }}$ ) scaled by the Einstein radius $\left(\theta_{\mathrm{E}}\right)$, and is therefore measurable from the resulting distortions of the lightcurve. It is a vector because these distortions depend on the direction of lens-source relative motion $(\boldsymbol{\mu})$ relative to the ecliptic. Because microlensing events are typically short compared to Earth's orbital time, these effects are not usually large. However, disk lenses are an important exception because for them $\pi_{\text {rel }}$ (and so $\pi_{\mathrm{E}}$ ) can be big. In particular, $\pi_{\mathrm{E}, \|} \equiv \hat{\mathbf{n}}_{a} \cdot \boldsymbol{\pi}_{\mathrm{E}}$, the component of $\boldsymbol{\pi}_{\mathrm{E}}$ parallel to Earth's instantaneous direction of acceleration projected on the sky, $\hat{\mathbf{n}}_{a}$, is usually much better measured than $\pi_{\mathrm{E}, \perp}$ (Gould et al. 1994; Smith et al. 2003; Gould 2004). This is because lens-source motion in this direction leads to an asymmetric distortion in the lightcurve, which is not easily confused with other microlensing effects. Note from Equation (6) that $\boldsymbol{\pi}_{\mathrm{E} \text {,hel }}$ and $\boldsymbol{\pi}_{\mathrm{E} \text {,geo }}$ have the same amplitude but different directions. It is actually $\pi_{\mathrm{E}, \| \text {,geo }}$ that is well-measured in microlensing events.

The first point is that if $\boldsymbol{\pi}_{\mathrm{E} \text {,geo }}$ is well measured, then one can determine the projected velocity in the geocentric frame $\tilde{\mathbf{v}}_{\text {geo }}=\boldsymbol{\pi}_{\mathrm{E} \text {,geo }}\left(\mathrm{AU} / \pi_{\mathrm{E}}^{2} t_{\mathrm{E}}\right)$ and so solve for it in the heliocentric frame $\tilde{\mathbf{v}}_{\text {hel }}=\tilde{\mathbf{v}}_{\text {geo }}+\mathbf{v}_{\oplus, \perp}$. Then, since $\mu / \tilde{v}=\pi_{\text {rel }} / \mathrm{AU}$, one can solve directly for $\boldsymbol{\mu}_{\text {geo }}$ and so $\theta_{*}=\mu_{\text {geo }} t_{*}$,

$$
\boldsymbol{\mu}_{\text {geo }}=\boldsymbol{\mu}_{\text {hel }}-\mu_{\text {hel }}\left|\frac{\boldsymbol{\pi}_{\mathrm{E}, \text { geo }}}{\pi_{\mathrm{E}}^{2} t_{\mathrm{E}}}+\boldsymbol{\mu}_{\oplus}\right|^{-1} \boldsymbol{\mu}_{\oplus}
$$

To understand the role of microlens parallax measurements more generally, I write $\boldsymbol{\mu}_{\text {geo }}$ in terms of observables.

$$
\boldsymbol{\mu}_{\text {geo }}=\boldsymbol{\mu}_{\text {hel }}-\theta_{\mathrm{E}} \pi_{\mathrm{E}} \boldsymbol{\mu}_{\oplus}=\boldsymbol{\mu}_{\mathrm{hel}}-\mu_{\text {geo }} t_{\mathrm{E}} \pi_{\mathrm{E}} \boldsymbol{\mu}_{\oplus}
$$

where I have written $\theta_{\mathrm{E}}=\mu_{\text {geo }} t_{\mathrm{E}}$ in the final step, since $t_{\mathrm{E}}$ is evaluated during the event, i.e., in the geocentric frame.

As pointed out by Ghosh et al. (2004), a good mea-

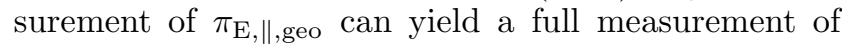
$\boldsymbol{\pi}_{\mathrm{E}, \text { geo }}$ if one can extract the direction of $\boldsymbol{\pi}_{\mathrm{E} \text {,geo from }}$ late time astrometry of the lens and source after they have moved apart sufficiently to be separately resolved. However, what is actually measured from such data is $\boldsymbol{\mu}_{\text {hel }}$, and so the direction of $\boldsymbol{\pi}_{\mathrm{E} \text {,hel }}$, not of $\boldsymbol{\pi}_{\mathrm{E} \text {,geo }}$. This implies that such solutions must be sought selfconsistently. Depending on the angle between $\boldsymbol{\mu}_{\text {hel }}$ and $\boldsymbol{\mu}_{\oplus}$, this may be more or less difficult. Similarly, in the second form of Equation (8), $\mu_{\text {geo }}$ must also be solved self-consistently. Thus, the viability of this approach for disk lenses must be evaluated on a case by case basis, depending on the magnitude of the errors 
in the microlensing parallax vector and in the value of $\boldsymbol{\mu}_{\oplus} \cdot \boldsymbol{\mu}_{\text {hel }} / \mu_{\text {hel }}$ which determines the fractional amplitude of the correction. See Gould (2014) for a detailed discussion.

\subsection{Uncertainty of Surface Brightness Measurement}

The other elements going into the color/surfacebrightness calibration are the dereddened flux and color measurements $K_{0}$ and $(V-K)_{0}$. For a large fraction of current microlensing events, observations are routinely carried out in $V, I$, and $H$ using the ANDICAM camera on the $1.3 \mathrm{~m}$ CTIO-SMARTS telescope (DePoy et al. 2003), which employs an optical/infrared dichroic. This permits very precise measurement of the (reddened) source flux in these bands for highly magnified targets because the fractional photometric errors are small for bright targets and because the magnifications are known from the microlensing model. Usually there is a small correction from $H$ to $K$, which can be evaluated either using the spectral type (see below) or the late-time astrometric/photometric measurements when the source and lens are separated. The problem is to convert these measurements of $V$ and $K$ to $K_{0}$ and $(V-K)_{0}$.

The method would be the same as in current microlensing studies (offset from the RC) except that it would be carried out using a $[(V-K), K]$ colormagnitude diagram, rather than $[(V-I), I]$. These color errors are unknown at the present time but for sake of discussion I assume that they are similar to the current $(V-I)$ errors (particularly taking account of the fact that the spectrum will yield a temperature measurement). From Equation (1) this would contribute about $0.019 \mathrm{mag}$ error to the surface brightness measurement, which is similar to the effect of a $0.9 \%$ error in $\theta_{*}$. Hence, if the current $0.1 \mathrm{mag}$ error in the dereddened magnitude were not improved, this would be by far the largest error, equivalent to a $4.6 \%$ error in $\theta_{*}$. It is beyond the scope of the present paper to develop methods to improve this, but I note that since there have never been any systematic efforts to do so, it is an open question what might be achieved.

\subsection{Spectra}

In order to determine whether the color/surfacebrightness relation depends on metallicity, it is of course necessary to measure the metallicity, which can only be done reliably by taking a spectrum. For subgiants (also dwarfs), the only cost-effective way to obtain such spectra is during the microlensing event when the targets are highly magnified (e.g., Bensby et al. 2013 and references therein). For giant stars, it is practical (albeit more expensive) to obtain spectra after they have returned to baseline. Such spectra will automatically yield additional information, such as the temperature (which can refine the estimate of $\left.(V-K)_{0}\right)$ and the radial velocity (to help identify the host population).

\section{High Metallicity Targets}

Of the 56 microlensed dwarfs and subgiants observed by Bensby et al. (2013), 13 have best fit $[\mathrm{Fe} / \mathrm{H}]>0.3$. Of these, seven have measured finite source effects: MOA-2008-BLG-311, MOA-2008-BLG-310 (Janczak et al. 2010), MOA-2012-BLG-022, OGLE-2007-BLG-349 (Cohen et al. 2008, Dong et al., in prep), OGLE2012-BLG-0026 (Han et al. 2013), MOA-2010-BLG311S (Yee et al. 2013), and MOA-2011-BLG-278 (Shin et al. 2012b). Of these, four have effective temperatures $T_{\text {eff }}<$ 5500: OGLE-2007-BLG-349 (5237), OGLE2012-BLG-0026 (4815), MOA-2010-BLG-311 (5442), and MOA-2011-BLG-278 (5307).

I list these with their geocentric proper motions in Table 1, together with an estimate of their separation (assuming $\mu_{\text {hel }}=\mu_{\text {geo }}$ ) in 2024 when the Giant Magellan Telescope (GMT) is expected to be fully operational. The diffraction limit of GMT at $H$ band is about 17 mas.

I note that there are a number of red giants that have both $t_{*}$ measurements and archival spectra. Alcock et al. (1997) report $t_{*}=2.54$ days, for MACHO-95-30. From their reported $K_{0}=9.83$ and $(V-K)_{0}=5.03$ I derive $\theta_{*}=43 \mu \mathrm{as}$ and hence $\mu_{\text {geo }}=6.1 \mathrm{mas} \mathrm{yr}^{-1}$. They quote $T_{\text {eff }}=3700$ but do not attempt to derive a metallicity from their spectrum. EROS-2000BLG-5 has $[\mathrm{Fe} / \mathrm{H}]=-0.3, T_{\text {eff }}=4500$ (Albrow et al. 2001) and $t_{*}=0.48$ days and $\mu_{\mathrm{geo}}=5.0 \mathrm{mas} \mathrm{yr}^{-1}$ (An et al. 2002). For OGLE-2002-BLG-069, Cassan et al. (2006) report $T_{\text {eff }}=5000$ and $[\mathrm{Fe} / \mathrm{H}]=-0.6$, while Kubas et al. (2005) report $t_{*}=0.50$ days. I find $\mu_{\text {geo }}=5.0$ mas yr $^{-1}$ from my own notes for this event. Zub et al. (2011) report $T_{\text {eff }}=3667, t_{*}=1.25$ days, $\mu_{\text {geo }}=7.4$ mas yr$^{-1}$ for OGLE-2004-BLG-482, but do not attempt to estimate a metallicity from their spectrum of this cool $R \sim 40 R_{\odot} \mathrm{M}$ giant ${ }^{\mathbf{1}}$. Cassan et al. (2004) report $[\mathrm{Fe} / \mathrm{H}]=+0.3, T_{\text {eff }}=4250, t_{*}=$ 0.53 days, $\mu_{\text {geo }}=3.1 \mathrm{mas} \mathrm{yr}^{-1}$ for OGLE-2004-BLG254.

Thus, among these giants, the only one that both has a reported iron abundance and is metal rich is OGLE2004-BLG-254. There are other giants for which there are microlensed spectra. For example, Bensby et al. (2013) occasionally targetted giants (due to mistakes in my own photometric source classifications) but did not analyze these for their sample of "bulge dwarfs and subgiants". However, it is unknown which of these have measured $t_{*}$ and, in addition the metallicities are also unknown.

Finally, there are a substantial number of giants that undergo caustic crossings. As the new MOA-II and OGLE-IV surveys have come on line, with many survey observations per night, an increasing number of these yield measurable $t_{*}$. This number is likely to increase with the advent of the 3-telescope KMTNet survey in 2015. As noted above, it is feasible to take spectra of these giants at baseline (although more convenient

\footnotetext{
${ }^{1}$ Note that in text, Zub et al. (2011) actually report inconsistent numbers due to their use of " $\theta_{*}$ " for both source radius and diameter. The numbers quoted here are the correct ones.
} 
Table 1

High $[\mathrm{Fe} / \mathrm{H}]$ dwarfs and subgiants with measured $t_{*}$

\begin{tabular}{lcccc}
\hline \hline Event Name & {$[\mathrm{Fe} / \mathrm{H}]$} & $\begin{array}{c}T_{\text {eff }} \\
(\mathrm{K})\end{array}$ & $\begin{array}{c}\mu_{\text {geo }} \\
\left(\mathrm{mas} \mathrm{yr}^{-1}\right)\end{array}$ & $\begin{array}{c}\Delta \theta(2024) \\
(\mathrm{mas})\end{array}$ \\
\hline \hline MOA-2008-BLG-311 & $0.35 \pm 0.08$ & 5947 & 3.7 & 59 \\
MOA-2008-BLG-310 & $0.41 \pm 0.11$ & 5675 & 5.1 & 82 \\
MOA-2012-BLG-022 & $0.42 \pm 0.10$ & 5827 & 1.0 & 12 \\
OGLE-2007-BLG-349 & $0.42 \pm 0.26$ & 5237 & 3.1 & 53 \\
OGLE-2012-BLG-0026 & $0.50 \pm 0.44$ & 4815 & 3.7 & 44 \\
MOA-2010-BLG-311S & $0.51 \pm 0.19$ & 5442 & 7.1 & 99 \\
MOA-2011-BLG-278 & $0.52 \pm 0.39$ & 5307 & 4.0 & 52 \\
\hline \hline
\end{tabular}

when they are magnified). Hence, in principle one could assemble a substantial number with high metallicity for future measurement of $\mu_{\text {hel }}$ when they are sufficiently separated.

Indeed, Henderson et al. (2014) has assembled a catalog of 20 microlensing events with high proper motions $\left(\gtrsim 8\right.$ mas yr $^{-1}$ ) as determined from finite source effects, including 14 from the literature and six newly analyzed. Because of their high-proper motions, many of these may become suitable targets for proper motion measurements before GMT comes on line. I find that eight of these are giants and so suitable for obtaining post-event (unmagnified) spectra. These are MOA-2004-BGL35 (8 mas yr $\left.^{-1}\right)$, OGLE-2004-BLG-368 (8 mas yr $\left.{ }^{-1}\right)$, OGLE-2004-BLG-482 (8 mas yr $\left.^{-1}\right)$, OGLE-2006-BLG277 (13 mas yr$\left.^{-1}\right)$, MOA-2007-BLG-146 (10 as yr $\left.^{-1}\right)$, OGLE-2011-BLG-0417 (10 mas yr $\left.^{-1}\right)$, OGLE-2012BLG-0456 (12 mas yr $\left.^{-1}\right)$, and MOA-2013-BLG-029 $\left(9\right.$ mas $\left.^{-1} r^{-1}\right)$.

Note that, as mentioned above, there is already a spectrum of OGLE-2004-BLG-482 (Zub et al. 2011), but no metallicity measurement. Also note that OGLE2011-BLG-0417 is one of the very few microlensing events with a complete orbital solution for the binary lens (Shin et al. 2012a) and the only one for which the lens is bright enough to spectroscopically monitor for radial velocity (RV) varations. Gould et al. (2013) have therefore advocated an RV campaign in order to test whether the predictions of the microlensing model are correct. Since the lens RV is changing by several $\mathrm{km} \mathrm{s}^{-1}$ from epoch to epoch, while the source is not, it should be straight forward to remove this "foreground" and stack the resulting "decontaminated" spectra to obtain a deep spectrum of the source.

Of the 12 non-giant stars in the Henderson et al. (2014) catalog, two were observed by Bensby et al. (2013): OGLE-2012-BLG-0211 $([\mathrm{Fe} / \mathrm{H}]=-0.06)$ and MOA-2012-BLG-532 $([\mathrm{Fe} / \mathrm{H}]=-0.55)$.

Even the non-giants that lack spectra can be used to test the overall method outlined here. In this regard I note that most of the Henderson et al. (2014) sample have magnified $H$-band data from CTIO-SMARTS. The exceptions are MOA-2004-BLG35, MOA-2011-BLG-040, OGLE-2012-BLG-0456, and
MOA-2013-BLG-029, which completely lack such data, and MOA-2011-BLG-262 and MOA-2011-BLG-274, for which the $H$-band data are of less than top quality due to low magnification at the time they were taken.

Thus, the prospects are good for applying this technique to past and future microlensed sources and thereby calibrating the color/surface-brightness relation at high metallicity.

\section{ACKNOWLEDGMENTS}

This work was supported by NSF grant AST 1103471 and NASA grant NNX12AB99G.

\section{REFERENCES}

Albrow, M., An, J., Beaulieu, J.-P., et al. 2001, H $\alpha$ Equivalent Width Variations across the Face of a Microlensed K Giant in the Galactic Bulge, ApJL, 550, L173

Alcock, C., Allen, W. H., Allsman, R. A., et al. 1997, MACHO Alert 95-30: First Real-Time Observation of Extended Source Effects in Gravitational Microlensing, ApJ, 491,436

An, J., Albrow, M., Beaulieu, J.-P., et al. 2002, First Microlens Mass Measurement: PLANET Photometry of EROS BLG-2000-5, ApJ, 572, 521

Anderson, E., \& Francis, C. 2012, XHIP: An Extended Hipparcos Compilation, Astronomy Letters, 38, 331

Batista, V., Beaulieu, J.-P., Gould, A., et al. 2014, MOA2011-BLG-293Lb: First Microlensing Planet Possibly in the Habitable Zone, ApJ, 780, 54

Bensby, T., Yee, J. C., Feltzing, S., et al. 2013, Chemical Evolution of the Galactic Bulge as Traced by Microlensed Dwarf and Subgiant Stars. V. Evidence for a Wide Age Distribution and a Complex MDF, A\&A, 549, 147

Bessell, M. S., \& Brett, J. M. 1988, JHKLM Photometry - Standard Systems, Passbands, and Intrinsic Colors, PASP, 100, 1134

Cassan, A., Beaulieu, J.-P., Brillant, S., et al. 2004, Probing the Atmosphere of the Bulge G5III Star OGLE-2002BUL-069 by Analysis of Microlensed $\mathrm{H} \alpha$ Line, A\&A, 419, 1

Cassan, A., Beaulieu, J.-P., Fouqué, P., et al. 2006, OGLE 2004-BLG-254: a K3 III Galactic Bulge Giant Spatially Resolved by a Single Microlens, A\&A, 460, 277

Cohen, J. G., Huang, W., Udalski, A., Gould, A., \& Johnson, J. A. 2008, Clues to the Metallicity Distribution in the Galactic Bulge: Abundances in OGLE-2007-BLG349S, ApJ, 682, 1029 
DePoy, D. L., Atwood, B., Belville, S. R., et al. 2003, A Novel Double Imaging Camera (ANDICAM), SPIE 4841, 827

Ghosh, H., DePoy, D. L., Gal-Yam, A., et al. 2004, Potential Direct Single-Star Mass Measurement, ApJ, 615, 450

Gould, A. 2000, A Natural Formalism for Microlensing, ApJ, 542, 785

Gould, A. 2004, Resolution of the MACHO-LMC-5 Puzzle: The Jerk-Parallax Microlens Degeneracy, ApJ, 606, 319

Gould, A. 2014, Microlens Masses from 1-D Parallaxes and Heliocentric Proper Motions, JKAS, submitted, arXiv1408.0797

Gould, A., Miralda-Escudé, J., \& Bahcall, J. N. 1994, Microlensing Events: Thin Disk, Thick Disk, or Halo?, ApJL, 423, L105

Gould, A., Shin, I.-G., Han, C., Udalski, A., \& Yee, J. C. 2013, OGLE-2011-BLG-0417: A Radial Velocity Testbed for Microlensing, ApJ, 768, 126

Han, C., Udalski, A., Choi, J.-Y., et al. 2013, The Second Multiple-Planet System Discovered by Microlensing: OGLE-2012-BLG-0026Lb,c -A Pair of Jovian Planets beyond the Snow Line, ApJL, 762, L28

Henderson, C. B., Park, H., Sumi, T., et al. 2014, Candidate Gravitational Microlensing Events for Future Direct Lens Imaging, ApJ, submitted. arXiv1403.3092

Janczak, J., Fukui, A., Dong, S., et al. 2010, Sub-Saturn Planet MOA-2008-BLG-310Lb: Likely to be in the Galactic Bulge, ApJ, 711, 731

Kervella, P., Thévenin, F., Di Folco, E., \& Ségransan, D. 2004, The Angular Sizes of Dwarf Stars and Subgiants. Surface Brightness Relations Calibrated by Interferometry, A\&A, 426, 297

Kubas, D., Cassan, A., Beaulieu, J.-P., et al. 2005, Full Characterization of Binary-Lens Event OGLE-2002BLG-069 from PLANET Observations, A\&A, 435, 841

Nataf, D. M., Gould, A., Fouqué, P., et al. 2013, Reddening and Extinction toward the Galactic Bulge from OGLEIII: The Inner Milky Way's $R_{\mathrm{V}} \sim 2.5$ Extinction Curve, ApJ, 769, 88

Richichi, A., Percheron, I., \& Khristoforova, M. 2005, CHARM2: An updated Catalog of High Angular Resolution Measurements, A\&A, 431, 773

Shin, I.-G., Han, C., Choi, J.-Y., et al. 2012, Characterizing Low-Mass Binaries from Observation of LongTimescale Caustic-Crossing Gravitational Microlensing Events, ApJ, 755, 91

Shin, I.-G., Han, C., Gould, A., et al. 2012, Microlensing Binaries with Candidate Brown Dwarf Companions, ApJ, 760,116

Smith, M. C., Mao, S., \& Paczyński, B. 2003, Acceleration and Parallax Effects in Gravitational Microlensing, MNRAS, 339, 925

Yee, J. C., Svartzvald, Y., Gal-Yam, A., et al. 2012, MOA2011-BLG-293Lb: A Test of Pure Survey Microlensing Planet Detections, ApJ, 755, 102

Yee, J. C., Hung, L.-W., Bond, I. A., et al. 2013, MOA-2010BLG-311: A Planetary Candidate below the Threshold of Reliable Detection, ApJ, 769, 77

Yoo, J., DePoy, D. L., Gal-Yam, A., et al. 2004, OGLE2003-BLG-262: Finite-Source Effects from a Point-Mass Lens, ApJ, 603, 139

Zub, M., Cassan, A., Heyrovský, D., et al. 2011, LimbDarkening Measurements for a Cool Red Giant in Microlensing Event OGLE 2004-BLG-482, A\&A, 525, A15 